\title{
Displacement Operators and Constraints on Boundary Central Charges
}

\author{
Christopher Herzog, ${ }^{1}$ Kuo-Wei Huang, ${ }^{1,2}$ and Kristan Jensen ${ }^{3}$ \\ ${ }^{1}$ C. N. Yang Institute for Theoretical Physics, Stony Brook University, Stony Brook, New York 11794, USA \\ ${ }^{2}$ Perimeter Institute for Theoretical Physics, Waterloo, Ontario N2L 2Y5, Canada \\ ${ }^{3}$ Department of Physics and Astronomy, San Francisco State University, San Francisco, California 94132, USA
}

(Received 2 October 2017; published 8 January 2018)

\begin{abstract}
Boundary conformal field theories have several additional terms in the trace anomaly of the stress tensor associated purely with the boundary. We constrain the corresponding boundary central charges in threeand four-dimensional conformal field theories in terms of two- and three-point correlation functions of the displacement operator. We provide a general derivation by comparing the trace anomaly with scale dependent contact terms in the correlation functions. We conjecture a relation between the $a$-type boundary charge in three dimensions and the stress tensor two-point function near the boundary. We check our results for several free theories.
\end{abstract}

DOI: 10.1103/PhysRevLett.120.021601

There is a strong argument for considering, from an abstract point of view, boundaries in quantum field theory (QFT). Boundary effects can be seen as a unifying theme in several areas where there has been enormous progress in theoretical physics. They are essential to understanding condensed matter systems such as topological insulators and quantum impurity models. D-branes, i.e., the boundaries of fundamental strings, gave us nonperturbative insight into string theory and led to the second superstring revolution in the late 1990s. In gauge-gravity duality, which provides windows both on strongly interacting quantum field theories and on quantum gravity, quantum fields fluctuate on the conformal boundary of anti-de Sitter space. Entanglement entropy in field theory is usually defined with respect to spatial regions, introducing an "entangling" surface which separates the regions. Entanglement has given us new insight into renormalization group flow [1-3], and has deepened our understanding of black hole thermodynamics [4] and energy conditions [5].

Conformal field theories (CFTs) play a central role in QFT as fixed points of the renormalization group flow. It seems reasonable that boundary conformal field theories (bCFTs) should play a similarly central role in the study of QFT with a boundary. More specifically, given the important role of trace anomalies in CFT without a boundary, it is reasonable to expect that boundary terms in the trace anomaly should be important as well.

Published by the American Physical Society under the terms of the Creative Commons Attribution 4.0 International license. Further distribution of this work must maintain attribution to the author(s) and the published article's title, journal citation, and DOI. Funded by SCOAP ${ }^{3}$.
We begin with a general discussion of the boundary terms in the trace anomaly including definitions of the anomaly coefficients $a_{(3 \mathrm{D})}, b, b_{1}$, and $b_{2}$. We prove that the coefficients $b$ and $b_{2}$ are related to two- and three-point functions of the displacement operator. Our main results are Eqs. (11) and (20). We conjecture that the $a_{(3 \mathrm{D})}$ coefficient satisfies a related constraint [Eq. (23)], from which follows a lower bound [Ref. (24)] on $a_{(3 \mathrm{D})} / b$. We then demonstrate that our relations hold for free theories.

Trace anomalies and boundary central charges.-We are interested in a classically Weyl-invariant theory embedded in a curved spacetime with a smooth and compact codimension-one boundary. The quantization requires regularization which results in a nonvanishing expectation value of the stress tensor trace. The trace anomaly in a compact spacetime is well known [6]. In particular, there is no anomaly in odd dimensions. In the presence of a boundary, however, there are anomalies localized on the boundary, in both odd and even dimensions. These new anomalies have rich geometric structure and they introduce new central charges that could be used to characterize the theories.

Define the induced metric on the boundary as $h_{\mu \nu}=g_{\mu \nu}-$ $n_{\mu} n_{\nu}$, where $n_{\mu}$ is an outward-pointing normal vector. The extrinsic curvature is $K_{\mu \nu}=h_{\mu}^{\lambda} h_{\nu}^{\sigma} \nabla_{\lambda} n_{\sigma}$ where $\nabla_{\lambda}$ is the bulk covariant derivative. We denote the traceless part of the extrinsic curvature as $\hat{K}_{\mu \nu}=K_{\mu \nu}-\left(h_{\mu \nu} / d-1\right) K$, which transforms covariantly under the Weyl transformation, and it plays an important role in constructing boundary Weyl invariants.

In $d=3$ spacetime dimensions with a two-dimensional boundary, the anomaly only appears on the boundary, and it is given by [7]

$$
\left\langle T_{\mu}^{\mu}\right\rangle^{d=3}=\frac{\delta\left(x_{\perp}\right)}{4 \pi}\left(a_{(3 \mathrm{D})} \stackrel{\circ}{R}+b \operatorname{tr} \hat{K}^{2}\right),
$$


where $\delta\left(x_{\perp}\right)$ is a Dirac delta function with support on the boundary, and $\operatorname{tr} \hat{K}^{2}=\operatorname{tr} K^{2}-\frac{1}{2} K^{2} ; \stackrel{\circ}{R}$ is the boundary Ricci scalar. For free fields, the values of these boundary charges were computed in the literature [8-10]: $a_{(3 \mathrm{D})}^{s=0,(D)}=-\frac{1}{96}$, $a_{(3 \mathrm{D})}^{s=0,(R)}=\frac{1}{96}$ and $a_{(3 \mathrm{D})}^{s=(1 / 2)}=0$, where $(D)$ and $(R)$ represent the Dirichlet and Robin boundary conditions, respectively boundary condition. (In our notation, $s$ is the spin of the free field.) The $a_{(3 \mathrm{D})}$ coefficient has been argued to decrease under boundary renormalization group flow [8].

The structure becomes much richer in $d=4$ CFTs. The complete classification was recently given in Ref. [11]. Dropping a regularization dependent term, the trace anomaly reads

$$
\begin{aligned}
\left\langle T_{\mu}^{\mu}\right\rangle^{d=4}= & \frac{1}{16 \pi^{2}}\left(c W_{\mu \nu \lambda \rho}^{2}-a_{(4 \mathrm{D})} E_{4}\right) \\
& +\frac{\delta\left(x_{\perp}\right)}{16 \pi^{2}}\left(a_{(4 \mathrm{D})} E_{4}^{(\mathrm{bry})}-b_{1} \operatorname{tr} \hat{K}^{3}-b_{2} h^{\alpha \gamma} \hat{K}^{\beta \delta} W_{\alpha \beta \gamma \delta}\right),
\end{aligned}
$$

where $E_{4}$ is the bulk Euler density in $d=4$, and $W_{\mu \nu \rho \sigma}$ is the Weyl tensor. In the presence of a boundary, the boundary term of the Euler characteristic, $E^{(\text {bry })}$, is added in order to preserve the topological invariance. We refer readers to the literature for the values of the $a_{(4 \mathrm{D})}$ and $c$ charges; these are the familiar central charges characterizing theories on a compact manifold. Let us list the values of the $b_{1}$ charge for free fields: $b_{1}^{s=0,(D)}=\frac{2}{35}$ [12], $b_{1}^{s=0,(R)}=\frac{2}{45}[13], b_{1}^{s=(1 / 2)}=\frac{2}{7}[10], b_{1}^{s=1}=\frac{16}{35}[10]$.

References $[14,15]$ observed that a relation $b_{2}=8 c$ is universal for free theories. Such a relation can be better understood by studying the stress tensor two-point function carefully with a boundary. Two of us have argued [16] that the relation need not hold once interactions are included. For a theory with a line of fixed points, parametrized by $g$, we found that, perturbatively in the coupling, $b_{2}=8 c+O\left(g^{2}\right)$. Note that Wess-Zumino consistency implies that $a$-type central charges cannot depend on marginal couplings [17].

The motivation of this Letter is to generalize Ref. [16] to consider other boundary charges in $d=3$ and $d=4$ CFTs. It turns out that the general strategy is similar: one simply looks at the correlation functions of the displacement operator in flat space. But there are several differences when compared with the computation of the $b_{2}$ charge. The first difference is that these $b$ and $b_{1}$ boundary charges do not talk to bulk charges, while the $b_{2}$ structure is intimately related to the surface term generated from varying the bulk $c$-type anomaly effective action. The second difference is that in order to compute $b_{1}$ in $d=4$, one has to look not at two-point functions but at a boundary three-point function. We will conjecture a relation for $a_{(3 \mathrm{D})}$, in terms of a boundary limit of the two-point function of the stress tensor.
Displacement operator and general relations.-To set the notation, let $W$ be the generating functional for connected Green's functions. The stress tensor in Euclidean signature is

$$
\left\langle T_{\mu \nu}(x)\right\rangle=-\frac{2}{\sqrt{g}} \frac{\delta W}{g^{\mu \nu}(x)} .
$$

Let us first consider $d=3$ CFTs with a boundary. Denote $\tilde{W}$ as the anomalous part of $W$. The anomaly effective action in dimensional regularization is

$$
\tilde{W}=\frac{\mu^{\epsilon}}{\epsilon} \frac{1}{4 \pi}\left(a_{(3 \mathrm{D})} \int_{\partial \mathcal{M}} \stackrel{\circ}{R}+b \int_{\partial \mathcal{M}} \operatorname{tr} \hat{K}^{2}\right) .
$$

Consider the special case where $\partial \mathcal{M}$ is almost the planar surface at $y=0$, and can be described by a small displacement $\delta y\left(x^{A}\right)$, which is a function of the directions tangent to the boundary, denoted by $x^{A}$. In this situation, the normal vector is well approximated by

$$
n_{\mu}=\left(\partial_{A} \delta y, 1\right) .
$$

The extrinsic curvature then becomes $K_{A B}=\partial_{A} \partial_{B} \delta y$, and we have

$$
\int_{\partial \mathcal{M}} \operatorname{tr} \hat{K}^{2}=\frac{1}{2} \int_{\partial \mathcal{M}} \delta y \stackrel{\circ}{\square}^{2} \delta y,
$$

where $\square^{2}=\partial^{A} \partial_{A}$ acts only on the boundary.

Correlation functions of the displacement operator $D^{n}(\mathbf{x})$ can be generated by varying $W$ with respect to $\delta y\left(x^{A}\right)$. Note that diffeomorphisms act on both the metric and the embedding function $\delta y\left(x^{A}\right)$. As the effective action $W$ is diffeomorphism invariant, there is a Ward identity that relates the stress tensor to the displacement operator, an integrated version of which in the flat limit becomes

$$
\left.T^{n n}\right|_{\partial \mathcal{M}}=D^{n} .
$$

Because the displacement operator lives inside the boundary surface, and because we have conformal symmetry in this surface, the two point function is fixed up to a constant, which we call $c_{n n}$ :

$$
\left\langle D^{n}(\mathbf{x}) D^{n}(0)\right\rangle=\frac{c_{n n}}{\mathbf{x}^{2 d}} .
$$

(In the notation of Ref. [16], $c_{n n}$ was called $\alpha(1)$ through its relation to the two point function of the stress tensor.) Replacing the expression Eq. (8) with a regularized version $[18,19]$ in the case of interest $d=3$,

$$
\left\langle D^{n}(\mathbf{x}) D^{n}(0)\right\rangle=\frac{c_{n n}^{(3 \mathrm{D})}}{512} \stackrel{\circ}{ }^{3}\left(\log \mu^{2} \mathbf{x}^{2}\right)^{2},
$$

the scale-dependent part is then 


$$
\mu \frac{\partial}{\partial \mu}\left\langle D^{n}(\mathbf{x}) D^{n}(0)\right\rangle=\pi \frac{c_{n n}^{(3 \mathrm{D})}}{32} \stackrel{\circ}{ }^{2} \delta(\mathbf{x}) .
$$

Equating the scale dependent pieces yields

$$
b=\frac{\pi^{2}}{8} c_{n n}^{(3 \mathrm{D})} .
$$

This relation was conjectured in Ref. [16], based on free theories $[8,10]$. Here we have provided a general derivation. A similar calculation for the case of a codimension-two defect in four dimensions was presented in Ref. [20] in the context of entanglement entropy. Note that the $b$ charge can change under marginal deformations, although here we do not discuss a 3D example.

Next we consider $d=4$. The constraint on the $b_{2}$ boundary charge was found in Ref. [16], and it reads

$$
b_{2}=\frac{2 \pi^{4}}{15} c_{n n}^{(4 \mathrm{D})} .
$$

In flat space, the two-point function is not enough to constrain the $b_{1}$ boundary charge, since the related Weyl anomaly has a $O\left(K^{3}\right)$ structure. Thus, we will need to consider the three-point function.

The relevant anomaly effective action is

$$
\tilde{W}^{\left(b_{1}\right)}=\frac{b_{1}}{16 \pi^{2}} \frac{\mu^{\epsilon}}{\epsilon} \int_{\partial \mathcal{M}} \operatorname{tr} \hat{K}^{3} .
$$

We again consider $\partial \mathcal{M}$ to be nearly flat and described by a small displacement, $\delta y\left(x^{A}\right)$. Approximating the normal vector by $n_{\mu}=\left(\partial_{A} \delta y, 1\right)$, we obtain

$$
\begin{aligned}
\int_{\partial \mathcal{M}} \operatorname{tr} \hat{K}^{3}= & \int_{\partial \mathcal{M}}\left(\operatorname{tr}\left[\left(\partial_{A} \partial_{B} \delta y\right)^{3}\right]\right. \\
& \left.-(\stackrel{\circ}{\square} \delta y) \operatorname{tr}\left[\left(\partial_{A} \partial_{B} \delta y\right)^{2}\right]+\frac{2}{9}(\stackrel{\circ}{\square} \delta y)^{3}\right) .
\end{aligned}
$$

We will relate this $b$ charge with the displacement operator three-point function defined by

$$
\left\langle D^{n}(\mathbf{x}) D^{n}\left(\mathbf{x}^{\prime}\right) D^{n}(\mathbf{0})\right\rangle=\frac{c_{n n n}}{|\mathbf{x}|^{4}\left|\mathbf{x}^{\prime}\right|^{4}\left|\mathbf{x}-\mathbf{x}^{\prime}\right|^{4}},
$$

where $c_{n n n}$ is a constant. The full structure of the stress tensor three-point function with a boundary has not been studied yet. But, as mentioned earlier, to constrain these boundary charges one can simply look at the purely normal-normal component of the stress-tensor correlation functions that represent the displacement operator contributions.

While it is not obvious how to proceed in position space, we note that the Fourier transform of the three-point function of operators $O_{1}, O_{2}$, and $O_{3}$ is generally [21,22]

$$
C_{123} \int_{0}^{\infty} d x x^{\alpha} \prod_{j=1}^{3} p_{j}^{\beta_{j}} K_{\beta_{j}}\left(p_{j} x\right),
$$

where $K_{\beta_{j}}(x)$ denotes the modified Bessel function of the second kind, and $\alpha=\frac{\delta}{2}-1, \beta_{j}=\Delta_{j}-\frac{\delta}{2} ; \Delta_{j}$ is the conformal dimension of operator $O_{j}$ and $\delta$ is the dimension of the CFT. In this case, we are interested in the CFT living on the boundary, so $\delta=3$ while the scaling dimension of the displacement operator is $\Delta_{j}=4$. Taking $c_{123}$ as the corresponding coefficient of the position space three-point function, one has [22]

$$
c_{n n n}=\frac{105}{\sqrt{2} \pi^{5 / 2}} C_{n n n} .
$$

The $1 / x$ term in a small $x$ expansion of the integrand will give rise to a logarithm in the position space three-point function and a corresponding anomalous scale dependence. Observe that the $1 / x$ term is

$$
\begin{aligned}
& \frac{3 \pi^{3 / 2}}{32 \sqrt{2} x}\left(p_{1}^{6}+p_{2}^{6}+p_{3}^{6}-p_{1}^{2} p_{2}^{4}-p_{1}^{2} p_{3}^{4}-p_{2}^{2} p_{1}^{4}\right. \\
& \left.-p_{2}^{2} p_{3}^{4}-p_{3}^{2} p_{1}^{4}-p_{3}^{2} p_{2}^{4}-\frac{2}{3} p_{1}^{2} p_{2}^{2} p_{3}^{2}\right) .
\end{aligned}
$$

Through integration by parts along the boundary, the above expression can be rewritten as

$$
\begin{gathered}
\frac{9 \pi^{3 / 2}}{4 \sqrt{2} x}\left(\left(p_{1} \cdot p_{2}\right)\left(p_{2} \cdot p_{3}\right)\left(p_{3} \cdot p_{1}\right)\right. \\
\left.-p_{1}^{2}\left(p_{2} \cdot p_{3}\right)^{2}+\frac{2}{9} p_{1}^{2} p_{2}^{2} p_{3}^{2}\right) .
\end{gathered}
$$

The result matches exactly the derivative form Eq. (14) computed from the $b_{1}$ boundary trace anomaly. Including a factor $(1 / 3$ !) coming from varying with respect to $\delta y$ three times, we obtain $b_{1}=(1 / 3 !) \times 16 \pi^{2}\left(9 \pi^{3 / 2} / 4 \sqrt{2}\right)\left(\sqrt{2} \pi^{5 / 2} / 105\right) c_{n n n}$, which gives

$$
b_{1}=\frac{2 \pi^{6}}{35} c_{n n n} .
$$

This boundary charge in $d=4$ can depend on marginal interactions. In particular, if the charge $b_{2}$ of the mixeddimensional quantum electrodynamics (QED) depends on the marginal interactions [16], so does $b_{1}$.

Conjecture for $a_{(3 \mathrm{D})}$.-From Refs. [16,23,24], we can write down expressions for the near-boundary limit of the stress-tensor two-point function:

$$
\left\langle T_{\mu \nu}(\mathbf{x}, y) T_{\rho \sigma}\left(\mathbf{0}, y^{\prime}\right)\right\rangle=A_{\mu \nu, \rho \sigma}\left(\mathbf{x}, y, y^{\prime}\right) \frac{1}{|\mathbf{x}|^{2 d}},
$$

where 


$$
\begin{aligned}
A_{n n, n n}\left(\mathbf{x}, y, y^{\prime}\right)= & \alpha(v) \\
A_{n A, n B}\left(\mathbf{x}, y, y^{\prime}\right)= & -\gamma(v) I_{A B}\left(\mathbf{x}, y, y^{\prime}\right) \\
A_{A B, C D}\left(\mathbf{x}, y, y^{\prime}\right)= & \alpha(v) \frac{d}{d-1} I_{A B, C D}^{(d)} \\
& +\left(2 \epsilon(v)-\frac{d}{d-1} \alpha(v)\right) I_{A B, C D}^{(d-1)}
\end{aligned}
$$

where $I_{A B}(x)=\delta_{A B}-2\left(x_{A} x_{B} / x^{2}\right)$ and $I_{A B, C D}^{(d)}=\frac{1}{2}\left(I_{A C} I_{B D}+\right.$ $\left.I_{A D} I_{B C}\right)-\frac{1}{d} \delta_{A B} \delta_{C D}$. The quantity $v$ is a cross-ratio $v=\left[\left(x-x^{\prime}\right)^{2} /\left(x-x^{\prime}\right)^{2}+4 y y^{\prime}\right]$, which behaves as $\sim 1-$ $\left(4 y y^{\prime} /|\mathbf{x}|^{2}\right)$ near the boundary at $v=1$.

The functions $\alpha, \gamma$, and $\epsilon$ are related to each other by two differential constraints. Conservation of the stress tensor at the boundary, conformal invariance, and unitarity together impose that $\gamma$ smoothly vanishes as $v \rightarrow 1$, while $\alpha$ is smooth, and $\epsilon$ can blow up as $(1-v)^{\delta-1}$ for a small anomalous dimension $\delta>0$. Both $\alpha$ and $\epsilon$ may have $O(1-v)^{0}$ terms, which we refer to as $\alpha(1)$ and $\epsilon(1)$. (Note the relation between $\alpha(v)$ and the $D^{n}$ two-point function, $\alpha(1)=c_{n n}$.)

The symmetries also allow for a boundary stress tensor which would only arise from decoupled boundary degrees of freedom. If present, it appears as a distributional term in the two-point function $C I_{A B, C D}^{(d-1)} \delta(y) \delta\left(y^{\prime}\right)$.

We conjecture that the boundary anomaly coefficient $a_{(3 \mathrm{D})}$ is a linear combination of $\alpha(1), \epsilon(1)$, and $C$. The dependence on $C$ is already fixed by the argument relating the trace anomaly of a two-dimensional CFT to the twopoint function of its stress tensor. More precisely, $c_{(2 \mathrm{D})}=2 \pi C$, where $c_{(2 \mathrm{D})}$ is the $2 \mathrm{D}$ central charge in the Euler anomaly $\left\langle T_{A}^{A}\right\rangle=\delta(y)\left[c_{(2 \mathrm{D})} / 24 \pi\right] \stackrel{\circ}{R}$. The coefficient $C$ vanishes for a theory of free $3 \mathrm{D}$ scalars and for free $3 \mathrm{D}$ fermions since these theories do not have extra decoupled boundary degrees of freedom. We fix the dependence on $\alpha(1)$ and $\epsilon(1)$ by the known values for the conformal scalar with Dirichlet and Robin boundary conditions, giving

$$
a_{(3 \mathrm{D})}=\frac{\pi^{2}}{9}\left(\epsilon(1)-\frac{3}{4} \alpha(1)+3 C\right) .
$$

Note this conjecture gives the correct result for free fermions, reproducing $a_{(3 \mathrm{D})}^{s=(1 / 2)}=0$.

In a general interacting bCFT we suspect only $\alpha(1)$ to be nonzero for the following reason. Interactions coupling boundary degrees of freedom to the bulk ought to lead to a unique stress tensor, leading to $C=0$. Meanwhile, $\epsilon(1)$ corresponds to a dimension- 3 boundary operator appearing in the boundary operator product expansion of $T_{A B}$, but the boundary conformal symmetry does not guarantee the existence of such an operator.

Reflection positivity means that the functions $\alpha(v)$ and $\epsilon(v)$ are non-negative [16]. The coefficient $C$ is also nonnegative. If $\epsilon(v)$ is regular near the boundary, then $\epsilon(1)$ is non-negative, and comparing with the new result [Eq. (11)] for $b$, we obtain the bound

$$
\frac{a_{(3 \mathrm{D})}}{b} \geq-\frac{2}{3}, \quad b \geq 0 \text { for } d=3 \text { bCFTs. }
$$

These bounds recall the Hofman-Maldacena [25] bounds on $d=4$ bulk central charges. However, if $\epsilon(v)$ is singular near the boundary, then there is no constraint on the sign of $\epsilon(1)$, and thus, no definite bound on $a_{(3 \mathrm{D})}$ charge. We note that $a_{(3 \mathrm{D})}$ and $b$ have been computed in a bottom-up holographic model [26] and their ratio falls below our proposed bound.

Two- and three-point functions in free theories.-We would like to verify the general relations Eqs. (11) and (20) in free theories, including a conformal scalar, a Dirac fermion and, in $d=4$, Maxwell theory.

The stress tensor two-point functions with a planar boundary for the scalar and fermion were already considered in Ref. [23]. More recently, Ref. [16] computed the two-point functions for a Maxwell field. We will list the relevant two-point function results for completeness, and consider three-point functions with a boundary in free theories. These latter results are, to our knowledge, new.

Considering first a vector of scalar fields, i.e., $\phi \rightarrow \phi^{a}$ (the index $a$ will be suppressed), we introduce complementary projectors $\Pi_{ \pm}$satisfying $\Pi_{+}+\Pi_{-}=\mathbb{1}$ and $\Pi_{ \pm}^{2}=\Pi_{ \pm}$. The boundary conditions are $\left.\partial_{n}\left(\Pi_{+} \phi\right)\right|_{y=0}=$ 0 and $\left.\Pi_{-} \phi\right|_{y=0}=0$. The scalar displacement operator is

$$
T_{n n}=\left(\partial_{n} \phi\right)^{2}-\frac{1}{4} \frac{1}{d-1}\left[(d-2) \partial_{n}^{2}+\square\right] \phi^{2},
$$

which is the boundary limit of the normal-normal component of the improved stress tensor. The two-point function of the scalar field can be found using the image method:

$$
\begin{aligned}
\left\langle\phi(x) \phi\left(x^{\prime}\right)\right\rangle= & \kappa\left(\frac{1}{\left|x-x^{\prime}\right|^{d-2}}\right. \\
& \left.+\frac{\chi}{\left(\left(\mathbf{x}-\mathbf{x}^{\prime}\right)^{2}+\left(y+y^{\prime}\right)^{2}\right)^{(d-2) / 2}}\right),
\end{aligned}
$$

where the parameter $\chi=\Pi_{+}-\Pi_{-}$is determined by boundary conditions. We have adopted the normalization $\kappa=$ $\left[1 /(d-2) \operatorname{Vol}\left(S^{d-1}\right)\right]$ where $\operatorname{Vol}\left(S^{d-1}\right)=\left[2 \pi^{(d / 2)} / \Gamma(d / 2)\right]$. Note $\chi^{2}=\mathbb{1}$, and that an eigenvalue of $\chi$ is 1 for Neumann and -1 for Dirichlet boundary conditions.

To keep the expressions simple, we will focus on the displacement operator two-point function in $d=3$ and the three-point function in $d=4$. These two quantities are required in computing the boundary central charges from the relations Eqs. (11) and (20).

A straightforward application of Wick's theorem gives

$$
\begin{gathered}
\left\langle D^{n}(\mathbf{x}) D^{n}(\mathbf{0})\right\rangle_{3 \mathrm{D}}^{s=0}=\frac{\operatorname{tr}(\mathbb{1})}{8 \pi^{2} \mathbf{x}^{6}}, \\
\left\langle D^{n}(\mathbf{x}) D^{n}\left(\mathbf{x}^{\prime}\right) D^{n}(\mathbf{0})\right\rangle_{4 \mathrm{D}}^{s=0}=\frac{1}{9 \pi^{6}} \frac{8 \operatorname{tr}(\mathbb{1})-\operatorname{tr}(\chi)}{|\mathbf{x}|^{4}\left|\mathbf{x}^{\prime}\right|^{4}\left|\mathbf{x}-\mathbf{x}^{\prime}\right|^{4}} .
\end{gathered}
$$


The result [Eq. (27)] implies that the $b$ boundary charge (in $d=3$ ) does not depend on boundary conditions for a free scalar. Indeed, using the relation Eq. (20), we recover the known value of the $b$ charge for a $d=3$ free scalar, $b=\frac{1}{64}$. On the other hand, clearly $b_{1}$ is sensitive to boundary conditions through the $\operatorname{tr}(\chi)$. Using the relation Eq. (20), we can verify that $b_{1}$ is $\frac{2}{35}$ for a Dirichlet scalar and $\frac{2}{45}$ for a Neumann scalar.

Next we consider a Dirac fermion. In Minkowski (mostly plus) signature, $\left\{\gamma_{\mu}, \gamma_{\nu}\right\}=-2 \eta_{\mu \nu}$. The fermion's displacement operator and two-point function are

$$
\begin{aligned}
T_{n n} & =\frac{i}{2}\left(\dot{\bar{\psi}} \gamma_{n} \psi-\bar{\psi} \gamma_{n} \dot{\psi}\right), \quad \dot{\psi} \equiv \partial_{n} \psi \\
\left\langle\psi(x) \bar{\psi}\left(x^{\prime}\right)\right\rangle & =\kappa_{f}\left(\frac{i \gamma \cdot\left(x-x^{\prime}\right)}{\left|x-x^{\prime}\right|^{d}}+\chi \frac{i \gamma \cdot\left(\bar{x}-x^{\prime}\right)}{\left|\bar{x}-x^{\prime}\right|^{d}}\right),
\end{aligned}
$$

where $\bar{x}=(-y, \mathbf{x})$ and $\kappa_{f}=1 / \operatorname{Vol}\left(S^{d-1}\right)$ and $\bar{\psi}=\psi^{\dagger} \gamma^{0}$. The $\chi$ parameter satisfies

$$
\chi \gamma_{n}=-\gamma_{n} \bar{\chi}, \quad \chi \gamma_{A}=\gamma_{A} \bar{\chi}, \quad \chi^{2}=\bar{\chi}^{2}=\mathbb{1},
$$

where $\bar{\chi}=\gamma^{0} \chi^{\dagger} \gamma^{0}$. Focusing on the fermion displacement operator two-point function in $d=3$ and the three-point function in $d=4$, we find

$$
\begin{gathered}
\langle D(\mathbf{x}) D(\mathbf{0})\rangle_{3 \mathrm{D}}^{s=(1 / 2)}=\frac{3}{16 \pi^{2}} \frac{\operatorname{tr}_{\gamma}(\mathbb{1})}{\mathbf{x}^{6}}, \\
\left\langle D(\mathbf{x}) D\left(\mathbf{x}^{\prime}\right) D(\mathbf{0})\right\rangle_{4 \mathrm{D}}^{s=(1 / 2)}=\frac{5}{4 \pi^{6}} \frac{\operatorname{tr}_{\gamma}(\mathbb{1})}{\mathbf{x}^{4} \mathbf{x}^{\prime 4}\left(\mathbf{x}-\mathbf{x}^{\prime}\right)^{4}},
\end{gathered}
$$

where $\operatorname{tr}_{\gamma}(\mathbb{1})$ depends on the Clifford algebra one uses; we will take $\operatorname{tr}_{\gamma}(\mathbb{1})=2^{\lfloor d / 2\rfloor}$. As $\chi^{2}=\mathbb{1}$, the boundary dependence drops out of these two- and three-point functions. We can again verify the relations Eqs. (11) and (20) for the fermion.

Finally, we consider a Maxwell field in Feynman gauge. As the field in $d=3$ is not conformal, we focus on the $d=4$ case. The displacement operator is

$$
T_{n n}=\frac{1}{2} F_{n A} F_{n}{ }^{A}-\frac{1}{4} F_{A B} F^{A B},
$$

and the gauge field two-point function is

$$
\left\langle A_{\mu}(x) A^{\nu}\left(x^{\prime}\right)\right\rangle=\kappa\left(\frac{\delta_{\mu}^{\nu}}{\left(x-x^{\prime}\right)^{2}}+\frac{\chi_{\mu}^{\nu}}{\left[\left(\mathbf{x}-\mathbf{x}^{\prime}\right)^{2}+\left(y+y^{\prime}\right)^{2}\right]^{2}}\right) .
$$

The $\chi_{\mu}^{\nu}$ parameter determines the boundary condition; it is equal to $\delta_{\mu}^{\nu}$ up to a sign. For gauge fields one can consider the absolute boundary condition where the normal component of the field strength is zero, which gives $\partial_{n} A_{A}=0$ and $A_{n}=0$, or the relative boundary condition where $A_{A}=0$ which gives $\partial_{n} A^{n}=0$ when recalling the gauge fixing. See Ref. [16] for more details. We find

$$
\left\langle D^{n}(\mathbf{x}) D^{n}\left(\mathbf{x}^{\prime}\right) D^{n}(\mathbf{0})\right\rangle_{4 \mathrm{D}}^{s=1}=\frac{512 \kappa^{3}}{|\mathbf{x}|^{4}\left|\mathbf{x}^{\prime}\right|^{4}\left|\mathbf{x}-\mathbf{x}^{\prime}\right|^{4}},
$$

independent of the choice of boundary conditions. From the relation Eq. (20) we recover the value of $b_{1}$ charge for the $d=4$ Maxwell field with a boundary.

Discussion.-We presented new results for the boundary terms in the trace anomaly for CFTs in 3D and 4D. By relating $b$ [Eq. (11)], $b_{1}$ [Eq. (20)], $b_{2}$ [Eq. (12)], and $a_{(3 \mathrm{D})}$ [Eq, (23)] to two- and three-point functions of the displacement operator and stress tensor in flat space, these results make the boundary coefficients more straightforward to compute.

While we proved the relations Eq. (11) and Eq. (20) in this Letter, two of us demonstrated Eq. (12) previously [16], and Eq. (23) remains a conjecture along with the lower bound Eq. (24) that follows from it (with the caveat discussed there). Ultimately, perhaps building on the bound Eq. (24), we hope that a classification scheme for bCFT can be organized around these coefficients. We suspect bounds on the 4D coefficients $b_{1}$ and $b_{2}$ exist as well, beyond $b_{2} \geq 0$ [16].

Finally, extending the $3 \mathrm{D}$ results to the case of a $4 \mathrm{D}$ bulk and 2D defect, there are applications of these results to quantum entanglement (see Ref. [20] for results along these lines).

We thank D. Gaiotto and R.-X. Miao for useful discussions. The work of C. P. H. and K.-W. H. was supported in part by the National Science Foundation under Grant No. PHY-1620628. The work of K. J. was supported in part by the U.S. Department of Energy under Grant No. DESC0013682.

[1] H. Casini and M. Huerta, A Finite entanglement entropy and the c-theorem, Phys. Lett. B 600, 142 (2004).

[2] H. Casini and M. Huerta, On the RG running of the entanglement entropy of a circle, Phys. Rev. D 85, 125016 (2012).

[3] H. Casini, E. Testé, and G. Torroba, Markov Property of the Conformal Field Theory Vacuum and the a Theorem, Phys. Rev. Lett. 118, 261602 (2017).

[4] H. Casini, Relative entropy and the Bekenstein bound, Classical Quantum Gravity 25, 205021 (2008).

[5] S. Balakrishnan, T. Faulkner, Z. U. Khandker, and H. Wang, A general proof of the quantum null energy condition, arXiv:1706.09432.

[6] S. Deser and A. Schwimmer, Geometric classification of conformal anomalies in arbitrary dimensions, Phys. Lett. B 309, 279 (1993).

[7] C. R. Graham and E. Witten, Conformal anomaly of submanifold observables in AdS/CFT correspondence, Nucl. Phys. B546, 52 (1999).

[8] K. Jensen and A. O'Bannon, Constraint on Defect and Boundary Renormalization Group Flows, Phys. Rev. Lett. 116, 091601 (2016). 
[9] M. Nozaki, T. Takayanagi, and T. Ugajin, Central charges for BCFTs and holography, J. High Energy Phys. 06 (2012) 066.

[10] D. V. Fursaev and S. N. Solodukhin, Anomalies, entropy and boundaries, Phys. Rev. D 93, 084021 (2016).

[11] C. P. Herzog, K.-W. Huang, and K. Jensen, Universal entanglement and boundary geometry in conformal field theory, J. High Energy Phys. 01 (2016) 162.

[12] J. Melmed, Conformal invariance and the regularised oneloop effective action, J. Phys. A 21, L1131 (1988).

[13] I. G. Moss, Boundary terms in the heat kernel expansion, Classical Quantum Gravity 6, 759 (1989).

[14] D. Fursaev, Conformal anomalies of CFT's with boundaries, J. High Energy Phys. 12 (2015) 112.

[15] S. N. Solodukhin, Boundary terms of conformal anomaly, Phys. Lett. B 752, 131 (2016).

[16] C. P. Herzog and K.-W. Huang, Boundary conformal field theory and a boundary central charge, J. High Energy Phys. 10 (2017) 189.

[17] H. Osborn, Weyl consistency conditions and a local renormalization group equation for general renormalizable field theories, Nucl. Phys. B363, 486 (1991).

[18] D. Z. Freedman, K. Johnson, and J. I. Latorre, Differential regularization and renormalization: A new method of calculation in quantum field theory, Nucl. Phys. B371 (1992) 353.
[19] H. Osborn and A.C. Petkou, Implications of conformal invariance in field theories for general dimensions, Ann. Phys. (N.Y.) 231, 311 (1994).

[20] L. Bianchi, M. Meineri, R. C. Myers, and M. Smolkin, Rényi entropy and conformal defects, J. High Energy Phys. 07 (2016) 076.

[21] A. Bzowski, P. McFadden, and K. Skenderis, Scalar 3-point functions in CFT: Renormalisation, beta functions and anomalies, J. High Energy Phys. 03 (2016) 066.

[22] A. Bzowski, P. McFadden, and K. Skenderis, Implications of conformal invariance in momentum space, J. High Energy Phys. 03 (2014) 111.

[23] D. M. McAvity and H. Osborn, Energy momentum tensor in conformal field theories near a boundary, Nucl. Phys. B406, 655 (1993).

[24] D. M. McAvity and H. Osborn, Conformal field theories near a boundary in general dimensions, Nucl. Phys. B455, 522 (1995).

[25] D. M. Hofman and J. Maldacena, Conformal collider physics: Energy and charge correlations, J. High Energy Phys. 05 (2008) 012.

[26] R.-X. Miao and C.-S. Chu, Universality for shape dependence of Casimir effects from Weyl anomaly, arXiv:1706 .09652 . 